\title{
The reliability of asleep-awake-asleep protocol for intraoperative functional mapping and cognitive monitoring in glioma surgery
}

\author{
Hugues Duffau
}

Received: 17 June 2013 / Accepted: 21 June 2013 / Published online: 14 July 2013

(C) Springer-Verlag Wien 2013

In this issue of Acta Neurochirurgica, Hansen et al. [9] proposed a new anaesthesiologic protocol for intrasurgical mapping "Awake craniotomies without any sedation: the awake-awake-awake technique". They reported a series of 50 patients who underwent such "awake-awake-awake" craniotomy for resection of 49 tumors (46 gliomas, three metastasis) and one cavernoma in eloquent areas. Patients did not require any sedation and no or only low-dose opioid treatment. The authors have therefore to be congratulated for their results.

Indeed, beyond the methodological aspect, the main message is that intraoperative electrical mapping should be universally implemented as standard of care for glioma surgery. This was demonstrated in a meta-analysis with 8091 adult patients who underwent resective surgery for supratentorial infiltrative glioma. It was shown that glioma resections using intrasurgical stimulation mapping were associated with fewer late severe neurologic deficits (less than $3.5 \%$ ) and more extensive resection, while they involved eloquent locations more frequently [3]. In this state of mind, awake surgery allows mapping of sensory-motor, visual, language and other cognitive functions, with a low rate of permanent neurologic worsening-less than $2 \%$ in the recent series $[4,7,10]$. This is the reason why the awake period should be optimal, with a perfect collaboration of the patient.

\section{H. Duffau $(\bowtie)$}

Department of Neurosurgery, Hôpital Gui de Chauliac,

Montpellier University Medical Center, 80 Av Augustin Fliche,

34295 Montpellier, France

e-mail: h-duffau@chu-montpellier.fr

\section{H. Duffau}

Institute of Neuroscience of Montpellier, INSERM U1051, Plasticity of Central Nervous System, Human Stem Cells and Glial Tumors, Hôpital Saint Eloi, CHU Montpellier, 80 Av Augustin Fliche, 34091 Montpellier, France
As a consequence, several issues may be discussed regarding the technical procedure advocated by Hansen et al. First of all, according to the authors, it seems that the classical "asleep-awake-asleep" technique has many drawbacks, side effects and risks. However, our team has recently published a prospective series with 140 patients who were operated on for a glioma involving eloquent area using "asleepawake-asleep" procedure, and demonstrated that no seizures, no swelling, no severe permanent neurological deficit and no mortality occurred (only one case of aspiration of gastric contents with a favorable outcome) [2]. These data confirmed the results we have already published in a previous retrospective study [4], demonstrating the reliability of "awake-asleep-awake" protocol. Nonetheless, in their experience, Hansen et al. [9] have reported a high rate of $16 \%$ of seizures using their technique, with $16 \%$ of new neurological deficits - which is a high rate in comparison with the recent literature (see above).

In addition, they have also claimed that patients undergoing awake craniotomies had "anxiety and fears", due to "terrifying noises and surroundings, immobility, loss of control, and the feeling of helplessness and being left alone". Yet, in a recent prospective European Low Grade Glioma Network multicenter study with 105 patients, awake-asleepawake surgery was used in the vast majority of cases (eight operations were performed according to an asleep-awake protocol, but never using awake-awake-awake technique) [1]. We demonstrated that awake surgery was well tolerated, as neither intraoperative nor postoperative assessment revealed major disadvantages. Especially, pain levels on a $10-\mathrm{cm}$ visual analogue scale were between $1.3 \mathrm{~cm}$ and $2.1 \mathrm{~cm}$, while levels of anxiety were between $2.2 \mathrm{~cm}$ and $2.6 \mathrm{~cm}$. In the same vein, Deras et al. observed only 0.7 of cooperation problem [2]. Thus, these findings demonstrate that asleep-awake-asleep protocol is also reproducible among institutions. 
Furthermore, this technique presents several advantages in comparison with the "awake-awake-awake" procedure. Firstly, there is no need for the patient to be awake during the opening, especially taking into account that this is the phase with maximum of "terrifying noise" (due to the craniotomy). Secondly, it is frequent to re-operate patients with glioma relapse. In these cases, the re-opening can be very difficult due to adherences, and it can take a long time. Once again, the participation of the patient is not useful during this period, whereas he has a risk to be tired during the awake phase - when the cooperation should be optimal to allow a reliable on-line cognitive monitoring (even if sometimes prolonged during several hours). Thirdly, in cases of voluminous tumors, in particular when dissection in the contact of the vessels can be required (e.g. fronto-temporo-insular gliomas), it seems more adapted that the patient sleeps again during the last part of the resection (in addition to the closure). Indeed, the awake electrostimulation mapping is necessary to define the functional boundaries, both at cortical and subcortical levels, in order to optimize the extent of resection, which should be continued up to critical structures [8], while preserving the quality of life [5]. However, once these limits (cortical epicenters and white matter pathways) have been reached, the part of the brain invaded by the glioma and now functionally disconnected can be removed without the help of the patient. In other words, the end of the resection (e.g. temporopolar or temporo-mesial resection, which may generate pain in the contact of the dura matter of the skull base; or dissection between tumor and arteries/veins, also sometimes painful in the contact of the middle cerebral artery; etc....) can be achieved under general anaesthesia [6]. This is more comfortable for patients and anaesthesiologists, especially when this second phase of resection takes a long time.

In summary, one should be reminded that, when a new technique is proposed, the goal is not to show its feasibility, but its usefulness. To this end, due to the lack of control group (asleep-awake-asleep) in the series by Hansen et al. [9], the authors did not clearly demonstrate the actual contribution of "awake-awake-awake" surgery in daily practice.
Maybe its interest might be investigated in more specific indications; for instance, in patients with obesity or clinically evident gastroesophagal reflux - that is, who can be considered as ineligible for asleep-awake-asleep procedure.

Conflicts of interest None.

\section{References}

1. Beez T, Boge K, Wager M, Whittle I, Fontaine D, Spena G, Braun S, Szelényi A, Bello L, Duffau H, Sabel M; for the European Low Grade Glioma Network (2013) Tolerance of awake surgery for glioma: a prospective European Low Grade Glioma Network multicenter study. Acta Neurochir (Wien). 2013 May 21. [Epub ahead of print]. PMID: 23689968 [PubMed - as supplied by publisher]

2. Deras P, Moulinié G, Maldonado IL, Moritz-Gasser S, Duffau H, Bertram L (2012) Intermittent general anesthesia with controlled ventilation for asleep-awake-asleep brain surgery: a prospective series of 140 gliomas in eloquent areas. Neurosurgery 71:764-771

3. de Witt Hamer PC, Gil Robles S, Zwinderman A, Duffau H, Berger MS (2012) Impact of intraoperative stimulation brain mapping on glioma surgery outcome: a meta-analysis. J Clin Oncol 30:2559 2565

4. Duffau H, Gatignol P, Mandonnet E, Capelle L, Taillandier L (2008) Intraoperative subcortical stimulation mapping of language pathways in a consecutive series of 115 patients with Grade II glioma in the left dominant hemisphere. J Neurosurg 109:461-471

5. Duffau H (2011) In: Duffau H (ed) Brain mapping: From neural basis of cognition to surgical applications. Springer, Wien

6. Duffau H (2012) A new concept of diffuse (low-grade) glioma surgery. Adv Tech Stand Neurosurg 38:3-27

7. Duffau H (2013) In: Duffau H (ed) Diffuse low-grade gliomas in adults natural history, interaction with the brain, and new individualized therapeutic strategies. Springer, London

8. Gil Robles S, Duffau H (2010) Surgical management of World Health Organization Grade II gliomas in eloquent areas: the necessity of preserving a margin around functional structures? Neurosurg Focus 28:E8

9. Hansen E, Seemann M, Zech N, Doenitz C, Luerding R, Brawanski A (2013) Awake craniotomies without any sedation: the awakeawake-awake technique. Acta Neurochir (Wien) 2013 [Epub ahead of print]. PMID: 23689969 [PubMed - as supplied by publisher]

10. Sanai N, Mirzadeh Z, Berger MS (2008) Functional outcome after language mapping for glioma resection. N Engl J Med 358:18-27 